\title{
NIL IDEALS OF POWER SERIES RINGS
}

\author{
EDMUND R. PUCZYROWSKI
}

(Received 2 October 1981; revised 25 March 1982)

Communicated by R. Lidl

\begin{abstract}
Some characterizations of nil radical and nil semisimple power series rings are given. The upper nil radical of a power series ring in an uncountable set of non-commutative indeterminates is completely described.
\end{abstract}

1980 Mathematics subject classification (Amer. Math. Soc.): 16 A 05, 16 A 21, 16 A 22.

\section{Introduction}

In this paper we investigate nil ideals of an associative power series ring $R\{X\}$ in a set $X$ of non-commutative indeterminates commuting with coefficients from $R$. We begin in Section 2 with a characterization of nil one-sided ideals of $R\{X\}$ for $X$ of cardinality $\geqslant 2$, showing that such ideals are contained in $N(R)\{X\}$, where $N(R)$ is the sum of all nilpotent ideals of $R$. It implies immediately that semiprime power series rings in non-commutative indeterminates are nil semisimple. This result and some of its applications have been obtained by the author (1980) for infinite $X$. A stronger result is proved in Section 3 for uncountable $X$. It asserts that a series $s$ belongs to a nil ideal of $R\{X\}$ if and only if the ideal of $R$ generated by the coefficients of $s$ is nilpotent. In the final section we present a result characterizing nil power series rings of one indeterminate.

All results of the paper are stated and proved for right ideals. By analogous arguments or using the fact that if $L$ is a nil left ideal of $A$ and $a \in L$ then the right ideal of $A$ generated by $a$ is nil, one can transfer all obtained results to left ideals.

(C) Copyright Australian Mathematical Society 1983 
The following simple observation will be very useful in our investigations.

LEMMA 1. The ideal $\langle A\rangle$ of $R$ generated by a subset $A$ is nilpotent if and only if for any denumerable subset $B$ of $R$ the set $A B$ is nilpotent.

Proof. If the ideal $\langle A\rangle$ is not nilpotent then for any $n=1,2, \ldots$ there exist elements $a_{n 1}, \ldots, a_{n n} \in A, b_{n_{1}}, \ldots, b_{n n} \in R$ such that $a_{n 1} b_{n 1} \cdots a_{n n} b_{n n} \neq 0$. Of course $B=\left\{b_{i j}\right\}$ is such a denumerable subset of $R$ that the set $A B$ is not nilpotent. This proves the part "only if". The part "if" is clear.

Throughout Sections 2 and $3, P(X)$ will be the free (non-abelian) semigroup with unity generated by the set $X$ of cardinality $\geqslant 2$. If $p \in P(X)$ and $x \in X$ then $l(p)$ and $l_{x}(p)$ will denote the degree and $x$-degree of $p$ respectively. The elements of $R\{X\}$ are the formal series $a=\Sigma a_{p} p$, where $a_{p} \in R, p \in P(X)$. For any $a \in R\{X\}$ we denote supp $a=\left\{p \in P(X) \mid a_{p} \neq 0\right\}$.

\section{Semisimplicity of power series rings}

We begin with an auxiliary lemma concerning the semigroup $P(X)$.

LEMMA 2. Let $x, y \in X, x \neq y$ and $Y=\left\{x y x, x y^{2} x, \ldots\right\} \subseteq P(X)$. Then

a) if $p, q \in P(X), r, t \in Y$ and $p r q=t$ then $p=q=1$;

b) if for $1 \leqslant i \leqslant n, p_{i}, q_{i} \in P(X), r_{i}, t_{i} \in Y, l\left(p_{i}\right) \geqslant l\left(q_{i}\right)$ and $p_{1} r_{1} \cdots p_{n} r_{n}=$ $q_{1} t_{1} \cdots q_{n} t_{n}$ then $p_{i}=q_{i}, r_{i}=t_{i}$ for $1 \leqslant i \leqslant n$.

Proof. The part a) follows immediately from the definition of $Y$. To prove b) we proceed by induction on $n$. If $n=1$ then $p_{1} r_{1}=q_{1} t_{1}$. But $l\left(p_{1}\right) \geqslant l\left(q_{1}\right)$, so $p_{1}=q_{1} s$ for some $s \in P(X)$. Thus $s r_{1}=t_{1}$ and by a) $s=1$. Hence $r_{1}=t_{1}$ and $p_{1}=q_{1}$.

Let us assume now that $b$ ) is valid for $n \leqslant k$ and $p_{1} r_{1} \cdots p_{k+1} r_{k+1}=q_{1} t_{1}$ $\cdots q_{k+1} t_{k+1}$. Let $p_{1}^{\prime}=p_{1} r_{1} p_{2}$ and $q_{1}^{\prime}=q_{1} t_{1} q_{2}$. We will show that $l\left(p_{1}^{\prime}\right) \geqslant l\left(q_{1}^{\prime}\right)$. Since $l\left(p_{1}\right) \geqslant l\left(q_{1}\right)$ then $p_{1}=q_{1}$ for some $s \in P(X)$. Thus

$$
s r_{1} p_{2} \cdots r_{k+1}=t_{1} q_{2} \cdots q_{k+1} t_{k+1} \text {. }
$$

By the assumption $l\left(q_{2}\right) \leqslant l\left(p_{2}\right)$, so if $l\left(p_{1}^{\prime}\right)<l\left(q_{1}^{\prime}\right)$ then $l\left(s r_{1}\right)<l\left(t_{1}\right)$. Now (l) implies that $s r_{1} w=t_{1}$ for some $w \in P(X)$. Hence by a) $s=w=1$ and, in consequence, $r_{1}=t_{1}$. This contradiction shows that $l\left(p_{1}^{\prime}\right) \geqslant l\left(q_{1}^{\prime}\right)$. Thus, using the induction argument, we obtain $r_{i}=t_{i}$ for $2 \leqslant i \leqslant k+1, p_{i}=q_{i}$ for $3 \leqslant i \leqslant k+1$ and $p_{1} r_{1} p_{2}=q_{1} t_{1} q_{2}$. The last equality and $l\left(p_{1}\right) \geqslant l\left(q_{1}\right), l\left(p_{2}\right) \geqslant l\left(q_{2}\right)$ give 
$p_{1}=q_{1} s$ and $p_{2}=w q_{2}$ for some $s, w \in P(X)$. Therefore $s r_{1} w=t_{1}$ and by a) $s=w=1$. This proves the lemma.

THEOREM 1. Let I be a nil right ideal of $R\{X\}$. If $a=\sum a_{p} p \in I$ then the ideal of $R$ generated by $A=\left\{a_{p} \mid p \in \operatorname{supp} a\right.$ has the minimal degree in supp $\left.a\right\}$ is nilpotent.

Proof. Let $b_{1}, b_{2}, \ldots \in R$ and $x, y \in X, x \neq y$. Using Lemma 2 we obtain that if $p_{i}$ for $1 \leqslant i \leqslant n$ have the minimal degree in supp $a$ then the coefficient at $p_{1} x y^{k_{1}} x \cdots p_{n} x y^{k_{n}} x$ in the series $\left(a\left(b_{1} x y x+b_{2} x y^{2} x+\cdots\right)\right)^{n}$ is equal to $a_{p_{1}} b_{k_{1}}$ $\cdots a_{p_{n}} b_{n_{n}}$. So if $\left(a\left(b_{1} x y x+b_{2} x y^{2} x+\cdots\right)\right)^{n}=0$ then $\left(A \cdot\left\{b_{i} \mid i=1,2, \ldots\right\}\right)^{n}=0$ and Lemma 1 ends the proof.

For any ring $R$ let $N(R)$ denote the sum of all nilpotent ideals of $R$. Similarly as for infinite $X$ (Puczylowski (1980), Corollary 3 ) we obtain

COROLlaRY 1. If $I$ is a nil right ideal of $R\{X\}$ and $a \in I$ then for any integer $k \geqslant 0$ the ideal of $R$ generated by $A_{k}=\left\{a_{p} \mid p \in \operatorname{supp} a, l(p)=k\right\}$ is nilpotent. In particular $I \subseteq N(R)\{X\}$.

Let $K$ be the nil radical class and $S$ the lower strong radical determined by $K$. It is well known (Divinsky, Krempa and Sulinski (1971)) that a ring $R$ is $S$-semisimple if and only if $R$ contains no non-zero nil right ideals. Thus by Theorem 1 we obtain immediately

COROLlaRY 2. For any ring $R$ the following conditions are equivalent:

(i) $R$ is semiprime;

(ii) $R\{X\}$ is semiprime;

(iii) $K(R\{X\})=0$;

(iv) $S(R\{X\})=0$.

\section{Special cases}

In this section we investigate nil ideals of $R\{X\}$ in some special cases. We start from a result on nil right ideals of bounded index.

Lemma 3. If $x, y \in X, x \neq y, p_{1}, \ldots, p_{n}, q_{1}, \ldots, q_{n} \in P(X)$, $\max \left(l\left(p_{1}\right), \ldots, l\left(p_{n}\right)\right)=k$ and

$$
p_{1} y x^{n(k+1)} \cdots p_{n} y x^{n(k+1)}=q_{1} y x^{n(k+1)} \cdots q_{n} y x^{n(k+1)}
$$

then $p_{1}=q_{1}, \ldots, p_{n}=q_{n}$. 
Proof. The equality (2) implies that for some $m \in P(X), q_{1}=p_{1} m$ or $p_{1}=$ $q_{1} m$. If $p_{1}=q_{1} m$ then $m y x^{n(k+1)} \cdots p_{n} y x^{n(k+1)}=y x^{n(k+1)} \cdots q_{n} y x^{n(k+1)}$. But if $m \neq 1$ - then $m=y x^{n(k+1)} r$ for some $r \in P(X)$. This is impossible as then $l\left(p_{1}\right)>n(k+1)>k$. If $q_{1}=p_{1} m$ then $y x^{n(k+1)} \cdots p_{n} y x^{n(k+1)}=m y x^{n(k+1)}$ $\cdots q_{n} y x^{n(k+1)}$. If $m \neq 1$ then $m=y x^{n(k+1)} r$ for some $r \in P(X)$. This implies that $l_{x}\left(q_{1}\right) \geqslant n(k+1)$ and, in consequence, $l_{x}\left(q_{1} y x^{n(k+1)} \cdots q_{n} y x^{n(k+1)}\right) \geqslant$ $n(n+1)(k+1)$. On the other hand $l_{x}\left(p_{1} y x^{n(k+1)} \cdots p_{n} y x^{n(k+1)}\right) \leqslant l\left(p_{1}\right)$ $+\cdots+l\left(p_{n}\right)+n^{2}(k+1) \leqslant k n+n^{2}(k+1)<n(n+1)(k+1)$, a contradiction.

THEOREM 2. If I is a nil right ideal of $R\{X\}$ satisfying the identity $a^{n}=0$ and $\langle A\rangle$ is the ideal of $R$ generated by $A=\left\{a_{p} \mid p \in \operatorname{supp} a, a \in I\right\}$ then $\langle A\rangle^{2 n}=0$ and, when $1 \in R,\langle A\rangle^{n}=0$.

Proof. Let $x, y \in X, x \neq y, a, \ldots, a_{n} \in A$ and $b_{1}, \ldots, b_{n} \in R$. The definition of $A$ implies that there exist $s_{1}, \ldots, s_{n} \in I$ and $q_{1} \in \operatorname{supp} s_{1}, \ldots, q_{n} \in \operatorname{supp} s_{n}$ such that $a_{i}$ is the coefficient at $q_{i}$ in $s_{i}$ for $i=1, \ldots, n$. Since for $i \neq j, 1 \leqslant i, j \leqslant n$, supp $s_{i} b_{i} x y^{i} x \cap \operatorname{supp} s_{j} b_{j} x y^{j} x=\varnothing$ then $a_{i} b_{i}$ is the coefficient at $p_{i}=q_{j} x y^{i} x$ in $s=s_{1} b_{1} x y x+\cdots+s_{n} b_{n} x y^{n} x$. Now if $k=\max \left(l\left(p_{1}\right), \ldots, l\left(p_{n}\right)\right)$ then by Lemma $3 a_{1} b_{2} \cdots a_{n} b_{n}$ is the coefficient at $p_{1} y x^{n(k+1)} \cdots p_{n} y x^{n(k+1)}$ in $\left(s y x^{n(k+1)}\right)^{n}$. But $s y x^{n(k+1)} \in I$, so $\left(s y x^{n(k+1)}\right)^{n}=0$. This shows that for any $a_{1}, \ldots, a_{n} \in A$, $b_{1}, \ldots, b_{n} \in R, a_{1} b_{1} \cdots a_{n} b_{n}=0$. Hence if $K$ is the right ideal of $R$ generated by $A R$ then $K^{n}=0$. But if $J$ is the right ideal of $R$ generated by $A$ then $J=K$ whenever $1 \in R$ and $J^{2} \subseteq K$ otherwise. This and the fact that indexes of nilpotency of $J$ and $\langle A\rangle$ are equal end the proof.

In particular Theorem 2 gives immediately

COROllary 3. An ideal I of $R\{X\}$ is nilpotent if and only if the ideal of $R$ generated by coefficients of all elements of I is nilpotent.

Now we will describe nil right ideals of $R\{X\}$ for uncountable $X$.

LEMMA 4. Let $a=\Sigma a_{p} p \in R\{X\}$. If $p_{1}, \ldots, p_{m}$ are such elements of supp $a$ that for some $x \in X,(a x)^{m}=0$ and $p_{1}, \ldots, p_{m} \in P(X \backslash\{x\})$, then $a_{p_{1}} \cdots a_{p_{m}}=0$.

Proof. Let us observe first that if $q_{1}, \ldots, q_{m} \in P(X)$ the equality $p_{1} x \cdots p_{m} x$ $=q_{1} x \cdots q_{m} x$ implies $p_{1}=q_{1}, \ldots, p_{m}=q_{m}$. Indeed, since $l_{x}\left(p_{1}\right)=\cdots=l_{x}\left(p_{m}\right)$ $=0$ then $l_{x}\left(q_{1}\right)+\cdots+l_{x}\left(q_{m}\right)+m=l_{x}\left(q_{1} x \cdots q_{m} x\right)=l_{x}\left(p_{1} x \cdots p_{m} x\right)=m$. Hence $l_{x}\left(q_{1}\right)=\cdots=l_{x}\left(q_{m}\right)=0$. Now the equality $p_{1} x \cdots p_{m} x=q_{1} x \cdots q_{m} x$ 
implies that for some $r \in P, p_{1}=q_{1} r$ or $q_{1}=p_{1} r$. But then $\operatorname{rxp}_{2} x \cdots p_{m} x=$ $x q_{2} x \cdots q_{m} x$ or $x p_{2} x \cdots p_{m} x=r x q_{2} x \cdots q_{m} x$. In both cases the condition $l_{x}(r)$ $=0$ implies that $r=1$, so $p_{1}=q_{1}$. Now $p_{2} x \cdots p_{m} x=q_{2} x \cdots q_{m} x$ and, analogously, we obtain that $p_{2}=q_{2}, \ldots, p_{2}=q_{2}, \ldots, p_{m}=q_{m}$. We conclude from the foregoing that the coefficient at $p_{1} x \cdots p_{m} x$ in the series $(a x)^{m}$ is equal to $a_{p_{1}} \cdots a_{p_{m}}$, so $a_{p_{1}} \cdots a_{p_{m}}=0$.

THEOREM 3. If the set $X$ is uncountable and $I$ is a nil right ideal of $R\{X\}$ then the ideal of $R$ generated by coefficients of an element of $I$ is nilpotent.

Proof. Let $x, y \in X, x \neq y$ and $b_{1}, b_{2}, \ldots \in R$. It can be easily seen that if $a=\Sigma a_{p} p \in I$ and $p \in \operatorname{supp} a$ then the coefficient at $p x y^{k} x$ in $a\left(b_{1} x y x+p_{2} x y^{2} x\right.$ $+\cdots)$ is equal to $a_{p} b_{k}$. The fact that $I$ is a nil right ideal of $R\{X\}$ implies that $\cup X_{n}=X$, where $X_{n}=\left\{z \in X \mid\left(a\left(b_{1} x y z+b_{2} x y^{2} x+\ldots\right) z\right)^{n}=0\right\}$. Thus for some $m$ the set $X_{m}$ is uncountable. In particular for any $p_{1}, \ldots, p_{m} \in \operatorname{supp} a$ and any natural numbers $k_{1}, \ldots, k_{m}$ there exists $z \in X_{m}$ such that $p_{1} x y^{k_{1}} x, \ldots, p_{m} x y^{k_{m}} x \in P(X \backslash\{z\})$. Hence from Lemma $4, a_{p_{1}} b_{k_{1}} \cdots a_{p_{m}} b_{k_{m}}=0$. Now Lemma 1 ends the proof.

COROLlaRY 4. If the set $X$ is uncountable then $S(R\{X\})=K(R\{X\})=$ $N(R\{X\})$.

Proof. Of course $S(R\{X\}) \supset K(R\{X\}) \supset N(R\{X\})$. By Theorem 3 the sum $W$ of all nil right ideals of $R\{X\}$ is equal to $N(R\{X\})$ so $W=N(R\{X\})=$ $K(R\{X\})$. Now if $I / K(R\{X\})$ is a nil right ideal of $R\{X\} / K(R\{X\})$ then $I$ is a nil right ideal of $R\{X\}$, so $I \subseteq W=K(R\{X\})$. Thus $R\{X\} / K(R\{X\})$ is $S$-semisimple. In consequence $S(R\{X\})=K(R\{X\})$.

\section{The case of one indeterminate}

It is known (Puczylowski (1980), Corollary 2) that if $X$ is a set of cardinality $\geqslant 2$ then $R\{X\}$ is nil if and only if $R$ is nilpotent or, equivalently, $R\{X\}$ is nilpotent. This is not true for power series rings $R\{x\}$ of one indeterminate $x$. Namely, let $P$ be the polynomial ring of commutative indeterminates $x_{1}, x_{2}, \ldots$ over a finite field of $p$ elements and let $I$ be the ideal of $P$ generated by $x_{1}^{p}, x_{2}^{p}, \ldots$. Since $P$ is a commutative algebra over a field of characteristic $p$ then for any $\Sigma a_{i_{1} \cdots i_{k}} x_{i_{1}}$ $\cdots x_{i_{k}} \in P, \quad\left(\sum a_{i_{1} \cdots i_{k}} x_{i_{1}} \cdots x_{i_{k}}\right)^{p}=\sum a_{i_{1} \cdots i_{k}}^{p} x_{i_{1}}^{p} \cdots x_{i_{k}}^{p} \in I$. Now if $\sum a_{i} x^{i} \in$ $(P / I)\{x\}$ then $\left(\sum a_{i} x^{i}\right)^{p}=\sum a_{i}^{p} x^{p i}=0$. Hence $(P / I)\{x\}$ is nil and $P / I$ is not nilpotent as for any $n, x_{1} \cdots x_{n} \notin I$. 
Gardner and Stewart (1976) have observed that the class $\bar{K}=\{R \mid R\{x\}$ is nil $\}$ is not radical. Now we will describe the class $\bar{K}$ more exactly.

THEOREM 4. Any ring of the class $\bar{K}$ is nil of bounded index.

Proof. If a ring $R$ is not nil of bounded index then for any $n=1,2, \ldots$ there exists $a_{n} \in R, a_{n}^{n} \neq 0$. Let $\left\{k_{n}\right\}$ be the sequence of integers defined by induction as follows

$$
k_{1}=1, \quad k_{n+1}=n k_{n}+1 .
$$

Certainly if $l>n$ then $k_{l} \geqslant k_{n+1}=n k_{n}+1$. Thus if $k_{i_{1}}+\cdots+k_{i_{n}}=n k_{n}$ then $i_{r} \leqslant n$ for $1 \leqslant r \leqslant n$. But since $k_{i_{r}} \leqslant k_{n}$ and $k_{i_{r}}=k_{n}$ if and only if $i_{r}=n$, then the equality $k_{i_{1}}+\cdots+k_{i_{n}}=n k_{n}$ implies $i_{1}=\cdots=i_{n}=n$. Using this fact we obtain that the coefficient at $x^{n k_{n}}$ in the series $\left(\sum a_{i} x^{k_{i}}\right)^{n}$ is equal to $a_{n}^{n} \neq 0$. Thus the series $\sum a_{i} x^{k_{i}}$ is not nilpotent.

Remark. The Nagata-Higman Theorem (Jacobson (1964), page 274) and Theorem 4 imply that members of $\bar{K}$ which are algebras over a field of characteristic zero are nilpotent.

\section{References}

N. Divinsky, J. Krempa and A. Sulinski (1971), "Strong radical properties of alternative and associative rings," J. Algebra 17, 369-388.

B. J. Gardner and P. N. Stewart (1976), "Reflected radical classes," Acta Math. Acad. Sci. Hungar. 28, 293-298.

N. Jacobson (1964), Structure of rings (A. M. S. Colloquium Publications, Providence, R.I.).

E. R. Puczylowski (1980), "Radical of polynomial rings, power series rings and tensor products," Comm. Algebra 8, 1699-1709.

Institute of Mathematics

University of Warsaw

00-901 Warsaw

Poland 